\title{
ДЛЯ ОБОБЩЕННОЙ СТИЛТЬЕСОВСКОЙ СТРУНЫ ФУНКЦИЯ ВЛИЯНИЯ ПРОЩЕ И ЛУЧШЕ ФУНКЦИИ ГРИНА
}

\section{Ю. В. Покорный, Е. В. Гулынина, М. Б. Зверева}

В классе абсолютно непрерывных на отрезке $[0,1]$ функций рассматривается уравнение

$$
-u^{\prime}(x)+\int_{0}^{x} u d Q=F(x)-F(0), \quad 0<x<1,
$$

возникающее при описании малых плоских поперечных деформаций упругой струны, натянутой вдоль отрезка $[0,1]$, или малых продольных деформаций стержня, расположенного вдоль того же отрезка. Функции $Q(x)$ и $F(x)$ имеют ограниченные вариации. Исходя из физической, точнее - вариационной мотивации уравнения (1), мы даем точное описание функции влияния $K(x, \xi)$ для исходной системы, позволяющей выразить решение задачи (1) в традиционной для функции Грина форме

$$
u(x)=\int_{0}^{1} K(x, \xi) d F(\xi) .
$$

Подразумеваемые при определении функции влияния исходные физические условия на концах связываются нами со значениями скачков функций $Q$ и $F$. Уравнение (1) оказывается носителем всех классических свойств обыкновенных дифференциальных уравнений второго порядка, а функция влияния успешно исполняет роль традиционной функции Грина, хотя в данной ситуации традиционное для функции Грина описание через аксиоматику весьма затруднительно и, более того, нерентабельно, поскольку она чрезвычайно трудно обозрима.

В работе показывается, что функция влияния строго положительна внутри квадрата $0<x, s<1$.

1. Предтечей рассматриваемого уравнения (1) для нас послужили работы М. Г. Крейна и И. С. Каца для уравнения вида

$$
u_{+}^{\prime}(x)=u_{-}^{\prime}(0)-\lambda \int_{0}^{x+0} u d M,
$$

где функция $M(x)$ определяется распределением масс струны, $u_{+}^{\prime}(x)$ - правая производная. Интерес к этому уравнению авторы [1] мотивировали более ранними исследованиями Феллера [2], который в связи с задачей рассеяния (см. также комментарии в [3]) рассматривал уравнение

$$
-\frac{d}{d M(x)} u_{+}^{\prime}(x)=\lambda u(x), \quad 0 \leqslant x \leqslant 1 .
$$

У Феллера псевдопроизводная $d / d M(x)$ определялась напрямую пределом соответствующих конечноразностных отношений. У М.Г. Крейна и И. С. Каца уравнение (3) было внешним символом (2). В отличие от (1) в (2) фигурирует величина $u_{-}^{\prime}(0)$, которая вместе с $u_{+}^{\prime}(1)$ должна быть задана с уравнением (2) заранее, и это именуется поиском

Работа выполнена при финансовой поддержке гранта Минобразования РФ (КЦСПбГУ), грант № Е02-1.0-46, Российского фонда фундаментальных исследований, гранты № 04-01-00049, 02-01-00307, программы "Университеты России", проект УР.04.01.004, и гранта Президента РФ на поддержку ведущих научных школ № НШ-1643.2003.1.

(C) Ю. В. Покорный, Е. В. ГУлынина, М. Б. ЗверевА, 2006 
решения уравнения (2) в классе продленных функций. Числа $u_{-}^{\prime}(0)$ и $u_{+}^{\prime}(1)$ требовались, чтобы придать смысл скачкам производной в концевых точках. Ниже будет показано, что в присутствии в уравнении (2) этих чисел нет нужды. Заметим, что в работах Феллера и М. Г. Крейна для уравнений (2), (3) обсуждались достаточно простые свойства, не выше уровня теорем о перемежаемости. Точное описание уравнения типа (3) в терминах производных по мере проведено в [4]. Вопрос о построении аналога функции Грина в этих работах не обсуждался.

2. Пусть $F(x)$ - внешняя нагрузка, приложенная к исследуемому континууму на промежутке от 0 до $x$. И пусть $Q(x)$ определяет распределение упругой реакции внешней среды на том же промежутке. Предполагая натяжение струны единичным, выпишем функционал потенциальной энергии

$$
\Phi(u)=-\int_{(0,1)} \frac{u^{\prime 2}}{2} d x-\int_{[0,1]} \frac{u^{2}}{2} d Q+\int_{[0,1]} u d F,
$$

где через $u(x)$ обозначено смещение (деформация) точки струны от положения равновесия. Предположение об ограниченности вариаций $Q(x)$ и $F(x)$ естественно. Существенно отметить, что в правой части представления (4) интегралы участвуют по разным множествам. Первый - по интервалу $(0,1)$, исключая концы, что естественно, поскольку на концах собственных значений у производной $u^{\prime}(x)$ нет, и физически это означает, что в концевых точках у нас натяжение нулевое: струна растягивается в окрестности точек, но не в самих точках. А во втором и третьем слагаемом (4) существенно, что интегралы рассматриваются по отрезку $[0,1]$, включая концы, поскольку оба интеграла берутся по Стилтьесу, и это значит, что во втором и третьем слагаемых существенными оказываются скачки функций $Q$ и $F$ на концах

$$
\begin{aligned}
\Delta Q(0) & =Q(+0)-Q(0), & \Delta Q(1) & =Q(1)-Q(1-0), \\
\Delta F(0) & =F(+0)-F(0), & \Delta F(1) & =F(1)-F(1-0) .
\end{aligned}
$$

С помощью этих скачков мы можем все выражения в (4) свести к общему интегралу по интервалу $(0,1)$ с дополнительными внеинтегральными членами, а именно,

$$
\begin{aligned}
\int_{[0,1]} \frac{u^{2}}{2} d Q & =\int_{(0,1)} \frac{u^{2}}{2} d Q+\frac{u^{2}(0)}{2} \Delta Q(0)+\frac{u^{2}(1)}{2} \Delta Q(1), \\
\int_{[0,1]} u d F & =\int_{(0,1)} u d F+u(0) \Delta F(0)+u(1) \Delta F(1),
\end{aligned}
$$

что отразится и на формуле (4).

В дальнейшем всюду мы в качестве области определения функционала $\Phi$ будем рассматривать множество $E$ абсолютно непрерывных на $[0,1]$ функций, производные которых имеют ограниченную вариацию на $[0,1]$. Естественно, мы предполагаем, что $E$ содержит те функции, которые соответствуют реальным физическим деформациям, т.е. любой элемент $E$ удовлетворяет исходным физическим предположениям на концах исходного отрезка $[0,1]$.

Согласно принципу Ланранжа-Гамильтона реальная форма струны $u_{0}(x)$ должна являться минималью функционала (4). Первая вариация функционала (4) здесь находится по стандартной схеме Лагранжа. На минимали $u_{0}(x)$ она должна быть нулевой, что приводит к равенству

$$
\begin{aligned}
& -\int_{(0,1)} u_{0}^{\prime} h^{\prime} d x-\int_{(0,1)} u_{0} h d Q+\int_{(0,1)} h d F \\
& \quad+\left[\Delta F(0)-u_{0}(0) \Delta Q(0)\right] h(0)+\left[\Delta F(1)-u_{0}(1) \Delta Q(1)\right] h(1)=0 .
\end{aligned}
$$


Проинтегрировав первое слагаемое по частям, приведем равенство (5) к виду

$$
\begin{gathered}
\int_{(0,1)} h d\left(u_{0}^{\prime}-g+F\right)+\left[\Delta F(0)-u_{0}(0) \Delta Q(0)+u_{0}^{\prime}(0)\right] h(0) \\
+\left[\Delta F(1)-u_{0}(1) \Delta Q(1)-u_{0}^{\prime}(1)\right] h(1)=0
\end{gathered}
$$

где мы обозначили $d g(x)=u_{0}(x) d Q(x)$, т.е. $g(x)=\int_{+0}^{x} u_{0} d Q$ (обозначением нижнего предела в виде +0 мы подчеркиваем, что $Q$-мера точки $x=0$ здесь не учитывается, т.е. не учитывается возможный скачок $Q$ в нуле). Теперь в силу произвола $h$ имеем

$$
\int_{(0,1)} h d\left(u_{0}^{\prime}-g+F\right)=0
$$

и вдобавок два соотношения на концах

$$
\left[\Delta F(0)-u_{0}(0) \Delta Q(0)+u_{0}^{\prime}(0)\right] h(0)=0, \quad\left[\Delta F(1)-u_{0}(1) \Delta Q(1)-u_{0}^{\prime}(1)\right] h(1)=0 .
$$

Согласно естественному аналогу леммы Дюбуа-Реймонда, из (6) следует, что реальная форма струны $u_{0}(x)$ должна удовлетворять уравнению

$$
-u^{\prime}(x)+\int_{+0}^{x} u d Q=F(x)+\text { const. }
$$

Из (7) следует весьма примечательное обстоятельство. Для закрепленного конца, когда заранее предполагается, например, что $u(0)=0$ (или $u(1)=0$ ), вариации $h(x)$ в своем произволе должны удовлетворять на этом конце аналогичному условию $h(0)=0$ (или $h(1)=0)$, и потому соответствующее условие в (7) выполняется автоматически. В противном случае, если один из концов (или оба) жестко не закреплен, должно выполняться

$$
u(0) \Delta Q(0)-u^{\prime}(0)=\Delta F(0),
$$

или (для незакрепленного правого конца)

$$
u(1) \Delta Q(1)+u^{\prime}(1)=\Delta F(1) .
$$

Каждое из этих равенств естественно трактовать, как реализацию уравнения (8) в незакрепленном конце. Подчеркнем, что если струна изначально не закреплена на конце, то постановка какого-либо краевого условия в этом конце, как это обычно делается, исключена. Равенство (9) позволяет выразить в (8) константу, что дает вместо (8)

$$
-u^{\prime}(x)+\int_{0}^{x} u d Q=F(x)-F(0) .
$$

Заметим, что в отличие от (8), здесь мы учитываем (согласно (9)) возможные скачки $Q(x)$ и $F(x)$ в нуле.

3. Далее мы для определенности ограничимся рассмотрением случая струны с закрепленным правым и незакрепленным левым концом. Согласно предыдущему мы будем иметь для нее уравнение (1). В равенствах (8)-(10) последнее оказывается бессодержательным. Тем самым мы игнорируем влияние на правый конец скачка $F(x)$, т.е. сосредоточенной нагрузки. Иначе, что очевидно интуитивно, и быть не может - конец закреплен. Точно также и о влиянии $\Delta Q(1)$.

Заметим, что если $s \in(0,1)$ - точка разрыва функции $Q(x)$ или $F(x)$, то равенство $(1)$ при $x=s$ означает, что

$$
-u^{\prime}(s+0)+u^{\prime}(s-0)+u(s) \Delta Q(s)=\Delta F(s),
$$

где $u^{\prime}(s+0)$ означает правую производную $u_{+}^{\prime}(s)$, а $u^{\prime}(s-0)$ - левую производную $u_{-}^{\prime}(s)$. Если же в точке $s$ функции $Q(x)$ и $F(x)$ непрерывны, то с толкованием уравнения (1) не возникает никаких сложностей, так как производная $u^{\prime}(x)$ существует в обычном смысле и интеграл определяется однозначно. 
Теорема 1. При любом с в предположении монотонности $Q(x)$ и $F(x)$ уравнение (1) имеет на $[0,1]$ единственное решение, удовлетворяющее условию $u(1)=c$.

4. Обратимся к точному определению функции влияния, которое теперь можно сделать предельно корректным. На интуитивном уровне функция влияния $K(x, \xi)$ определяется как деформация исходной системы под влиянием единичной силы, приложенной к точке $\xi$. Для нас это означает, что $F(x)=\theta(x-\xi)$, где $\theta(x)$ - классическая функция Хевисайда, т.е. $\theta(x)=1$ при $x>0$ и $\theta(x)=0$ при $x<0$. Пользуясь вариационным описанием исследуемого объекта, мы определяем функцию влияния как решение $K(x, \xi)$ в $E$ уравнения (1) при $F(x)=\theta(x-\xi)$, т.е. уравнения

$$
-u^{\prime}(x)+\int_{0}^{x} u d Q=\theta(x-\xi) .
$$

Описанный подход снимает необходимость использовать аппарат теории обобщенных функций, поскольку мы обходим стандартную при определении функции Грина трудность опоры на фундаментальное решение как на обобщенное (по Шварцу-Соболеву) решение уравнения типа $L u=\delta(x-\xi)$. В рассматриваемом классе $E$ аналог такого решения у нас всегда имеется.

Замечательно то, что равенством (11) можно пользоваться поточечно, что позволяет описать важнейшие свойства функции влияния.

Teорема 2. 1) Если $\xi \neq 0, m o-K_{x}^{\prime}(+0, \xi)+K(0, \xi) \Delta Q(0)=0$.

2) $\Pi p u \xi=0-K_{x}^{\prime}(+0,0)+K(0,0) \Delta Q(0)=1$.

3) Если в точке $s \in(0,1)$ функиия $Q(x)$ непрерывна, то

$$
K_{x}^{\prime}(s+0, s)-K_{x}^{\prime}(s-0, s)=-1,
$$

а если s - точка разрыва $Q(x)$, то

$$
K_{x}^{\prime}(s+0, s)-K_{x}^{\prime}(s-0, s)-K(s, s) \Delta Q(s)=-1 .
$$

Теорема 3. Пусть функиия $Q(x)$ не убъвает на $[0,1]$, и пусть $Q \neq$ const. Тогда решение $K(x, \xi)$ уравнения (11) строго положительно на полуинтервале $[0,1)$, причем максимум решения достигается в точке $\xi \in[0,1)$ приложения импульса, т.е. $\max _{[0,1]} K(x, \xi)=K(\xi, \xi)$.

Теорема 4. Решение $и(x)$ уравнения (1) представимо в виде

$$
u(x)=\int_{0}^{1} K(x, \xi) d F(\xi) .
$$

В ходе доказательства теоремы устанавливается, что функция влияния является двупарным ядром, т.е. представима в виде

$$
K(x, \xi)=\frac{1}{\varphi_{2}(0)} \begin{cases}\varphi_{1}(x) \varphi_{2}(\xi), & 0 \leqslant \xi \leqslant x \leqslant 1, \\ \varphi_{2}(x) \varphi_{1}(\xi), & 0 \leqslant x \leqslant \xi \leqslant 1,\end{cases}
$$

где $\varphi_{1}(x)$ и $\varphi_{2}(x)$ - абсолютно непрерывные функции.

\section{СПИСОК ЦИТИРОВАННОЙ ЛИТЕРАТУРЫ}

[1] И. С. Кац, М. Г. Крейн, О спектральных функииях струны. Дополнение II к книге [3]. [2] W. Feller, J. Math., 1:4 (1957), 459-504. [3] Ф. Аткинсон, Дискретные и 
непрерывные граничные задачи, Мир, М., 1991. [4] Ю. В. Покорный, Докл. РАН, 383:5 (2002), 1-4.

Ю. В. Покорный

Поступило

Воронежский государственный университет

25.09.2005

E-mail: pokorny@kma.vsu.ru

Е. В. Гулынина

Северо-Кавказский филиал Белгородского государственного

технологического университета им. В. Г. Шухова, г. Минеральные

Воды

E-mail: belgtasm@mw.narzan.com

М. Б. Зверева

Воронежский государственный университет

E-mail: margz@rambler.ru 